\title{
Úlceras orales: diagnóstico diferencial y tratamiento
}

\author{
Oral ulcers: Differential diagnosis and treatment \\ Mayra Schemel-Suárez, José López-López y Eduardo Chimenos-Küstner* \\ Facultad de Odontología, Universidad de Barcelona
}

INFORMACIÓN DEL ARTÍCULO

\section{Historia del artículo:}

Recibido el 14 de marzo de 2015

Aceptado el 27 de abril de 2015

On-line el $\mathrm{xxx}$

\section{Introducción}

Las úlceras orales suelen ser muy dolorosas y son motivo de consulta frecuente. Entre sus causas destacan procesos infecciosos, neoplasias, enfermedades gastrointestinales, alteraciones sanguíneas, enfermedades reumatológicas, enfermedades inmunológicas, traumatismos y otros factores ${ }^{1,2}$. Debido a los diversos factores que pueden causarlas es necesario realizar un adecuado diagnóstico diferencial.

Las úlceras pueden ser primarias, cuando no están precedidas por otra lesión previa (es el caso de las aftas), o secundarias (aftoides) a un traumatismo o a la rotura de una ampolla o una vesícula ${ }^{3}$.

Las aftas, descritas por Hipócrates en el año 400 a. C., suelen localizarse en la mucosa oral (labios, lengua, suelo de la boca, paladar blando, úvula, etc.) y faríngea; se pueden observar también en la región genital ${ }^{4}$. La lesión primitiva no es ni una vesícula ni una ampolla, sino directamente la ulceración debida a una necrosis epitelial que sobrepasa la membrana basal, exponiendo terminaciones nerviosas lo que provoca molestias o dolor ${ }^{2,4}$. Si alcanzan gran tamaño, pueden dejar una cicatriz después de su curación. Clínicamente cursan como ulceraciones dolorosas, de tamaño variable, redondas u ovaladas y de bordes limpios. El fondo necrótico está cubierto por un exudado fibrinoso blanco-amarillento y su periferia está enmarcada por un ribete o halo rojo de origen hiperémico $\mathrm{y}$ tienen tendencia a la recidiva ${ }^{4}$. Las aftoides son úlceras secundarias, generalmente de origen infeccioso, que comienzan con una lesión fundamental: vesículas o vesículo-pústulas, clínica e histológicamente distintas a las aftas verdaderas. Otras aftoides son de origen traumático, de evolución aguda o crónica, como respuesta

\footnotetext{
* Autor para correspondencia.

Correos electrónicos: echimenos@ub.edu, eduardochimenoskustner@gmail.com (E. Chimenos-Küstner).
}

a un traumatismo reiterado y persistente de la mucosa. Algunas tienden a la cronicidad y pueden ser recidivantes (por ejemplo, la estomatitis herpética $)^{5}$. Las aftosis son determinados procesos que cursan con aftas o aftoides y generalmente con repercusión sistémica. En estos procesos las úlceras pueden localizarse en mucosa bucal y en otras como la genital, la anal o la conjuntival ${ }^{5}$.

\section{Diagnóstico diferencial}

El primer paso para realizar un correcto diagnóstico diferencial es la historia clínica. La anamnesis del paciente aporta datos esenciales para identificar el tipo de lesión que este presenta. Se deben investigar los antecedentes médicos (antecedentes familiares y personales de enfermedad sistémica o dermatológica, fármacos, alergias, hábitos alimentarios y tóxicos $)^{1}$. Con el interrogatorio se debe intentar establecer: el momento de aparición de la primera lesión; el tiempo de evolución transcurrido o, si es recidivante, la duración de las lesiones; el área de la mucosa oral afecta; la coexistencia de lesiones similares o concomitantes en otras áreas (genitales, piel); la presencia de síntomas acompañantes presentan (dolor, fiebre, ardor); la asociación con algún traumatismo en la mucosa oral o con alguna otra situación (estrés, ciclo menstrual, cese del hábito tabáquico); y si hay alguna medicación que le alivie la sintomatología.

El examen clínico completará la anamnesis, mediante una inspección sistematizada de la mucosa oral. En este paso se debe tomar en cuenta el tipo de lesión (vesículas, ampollas, úlceras), número de lesiones, tamaño, apariencia, ubicación (mucosa queratinizada, mucosa no queratinizada o ambas), así como su relación con bordes o aristas cortantes de dientes o prótesis ${ }^{1}$.

Tras recoger todos estos datos se podrá establecer un diagnóstico presuntivo de la lesión, cuya confirmación puede requerir exámenes o procedimientos complementarios (analítica 


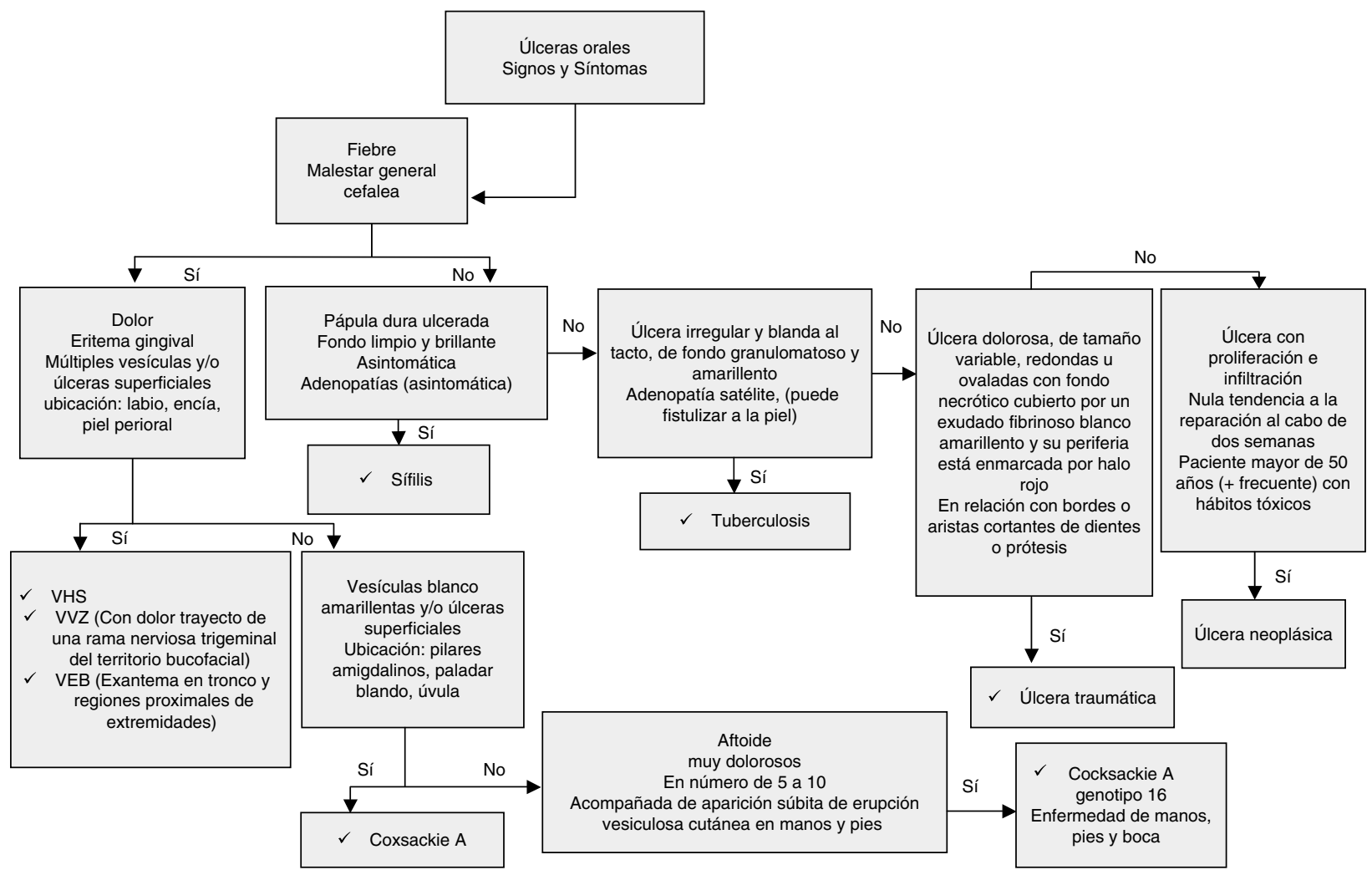

Figura 1. Diagrama para el diagnóstico clínico de las úlceras orales. VEB: virus de Epstein-Barr; VHS: virus del herpes simple; VVZ: virus varicela-zóster.

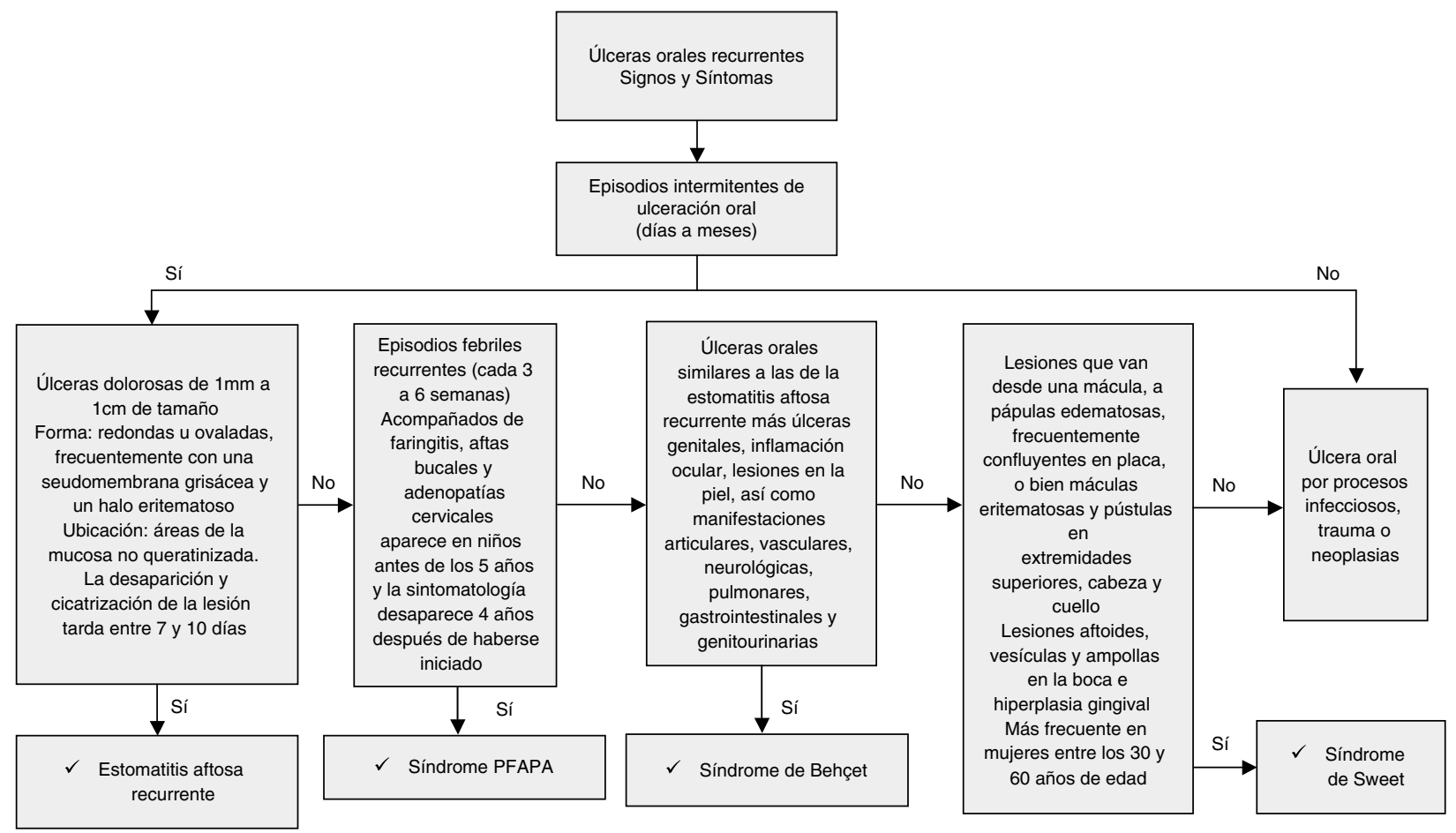

Figura 2. Diagrama para el diagnóstico clínico de las úlceras orales recurrentes. PFAPA: periodic fever, aphtae, pharyngitis y cervical adenopathies (fiebre periódica, aftas, faringitis y adenopatías cervicales). 
Tabla 1

Factores que pueden influir en la aparición de estomatitis aftosa recurrente

\begin{tabular}{|c|c|}
\hline Hereditarios & $\begin{array}{l}\text { La presencia de aftas en los padres influye significativamente en el riesgo de desarrollo de EAR en sus hijos }{ }^{17} \text {. Los factores genéticos de } \\
\text { riesgo incluyen varios polimorfismos en el ADN del genoma humano }{ }^{18}\end{array}$ \\
\hline Deficiencias nutricionales & $\begin{array}{l}\text { Niveles bajos en sangre de hierro, folato, cinc, o vitaminas } B_{1}, B_{2}, B_{6} \text { y } B_{12} \text {. Estas deficiencias pueden ser secundarias a otras } \\
\text { enfermedades, como el síndrome de malabsorción o la sensibilidad al gluten, asociada o no a enteropatías }{ }^{17}\end{array}$ \\
\hline Hormonales & $\begin{array}{l}\text { Los niveles de hormonas sexuales en sangre pueden exacerbar la frecuencia de EAR principalmente en la fase lútea del ciclo menstrual } \\
\text { y durante la menopausia, mientras que las remisiones aparecen frecuentemente durante el embarazo y en mujeres que toman } \\
\text { anticonceptivos }\end{array}$ \\
\hline Trauma local & $\begin{array}{l}\text { En individuos con predisposición a EAR las lesiones aparecerán en la mucosa oral poco después de la irritación mecánica del área }{ }^{6} \text {. } \\
\text { Existe baja incidencia de EAR en pacientes fumadores, esto puede ser debido a la hiperqueratinización de la mucosa oral en respuesta } \\
\text { al consumo de tabaco, haciéndola menos sensible a la irritación y al trauma }{ }^{19}\end{array}$ \\
\hline Estrés & El estrés desencadena los episodios de EAR pero no influye en su duración ${ }^{20}$ \\
\hline Infecciones & $\begin{array}{l}\text { Se ha observado que en pacientes infectados con Helicobacter pylori los síntomas de EAR son más intensos que en aquellos que no lo } \\
\text { están. La acción de la bacteria en la aparición y recurrencia de aftas estará asociada a anemia producida por enfermedades gástricas } \\
\text { positivas a Helicobacter pylori }{ }^{21} \text {. }\end{array}$ \\
\hline
\end{tabular}

EAR: estomatitis aftosa recurrente.

sanguínea $^{6}$, biopsia ${ }^{3,7}$ ). En las figuras 1 y 2 se recogen los principales cuadros causantes de úlceras orales, ante los que hay que plantearse el diagnóstico diferencial.

\section{Tratamiento}

En función del diagnóstico establecido y de acuerdo con los datos recogidos en la historia clínica, se planteará el tratamiento más adecuado. Prestamos especial atención a la terapia frente a las aftas, que destacan por ser las más frecuentes.

\section{Tratamiento de las aftoides}

En el caso de las úlceras de origen viral el tratamiento será sintomático (analgésicos y antipiréticos), para reducir la fiebre y controlar el dolor, ya que este tipo de lesiones tiene un corto período de duración y se resuelven espontáneamente. Es muy importante la ingesta adecuada de líquidos, especialmente en niños, y una dieta blanda en caso de que la masticación se vea limitada por el dolor ${ }^{2}$. También se pueden emplear, como tratamiento coadyuvante, los enjuagues con anestésicos locales ${ }^{8}$.

\section{Tratamiento de las úlceras específicas}

Si el diagnóstico presuntivo es el de una úlcera causada por Treponema pallidum se deberá realizar una biopsia incisional y una analítica sanguínea que incluya pruebas de VDRL, RPR y FTA-ABS. Si el diagnóstico resulta positivo, se deberá instaurar el tratamiento antibiótico indicado ${ }^{7}$. En el caso de una úlcera sospechosa de infección por Mycobacterium tuberculosis se tomará una biopsia incisional $^{9}$ y si el diagnóstico histopatológico es sugestivo de tuberculosis se deberá completar el estudio del paciente con los de exámenes complementarios adecuados (PPD, cultivo y radiografía de tórax), para que se pueda instaurar el tratamiento pertinente $^{10,11}$.

\section{Tratamiento de las úlceras traumáticas}

En las úlceras orales causadas por factores traumáticos, el tratamiento dependerá de la identificación del agente irritante y la eliminación de este (pulido de cúspides cortantes, ajuste de prótesis, técnicas de cepillado correcta, cambio de los productos de higiene bucal por unos menos irritantes, etc. $)^{5}$.

\section{Tratamiento de las úlceras neoplásicas}

Ante la sospecha de una úlcera neoplásica, se deberá realizar una biopsia incisional de la lesión; si el resultado histopatológico confirma el diagnóstico de sospecha de carcinoma de células escamosas, se derivará al paciente de inmediato a su centro de referencia, donde diferentes especialistas (oncólogos, cirujanos orales y maxilofaciales, radioterapeutas, quimioterapeutas, odontólogos, psicólogos y nutricionistas) proporcionarán el tratamiento más adecuado de acuerdo al estadio (TNM) en que se encuentre el tumor $^{12}$.

\section{Tratamiento de las aftas}

Estas suelen formar parte de un cuadro recidivante, conocido como estomatitis aftosa recurrente (EAR). Frente a este proceso patológico es recomendable realizar un análisis sanguíneo completo, que incluya hemograma, ácido fólico, ferritina y vitamina $B_{12}$, para descartar posibles causas sistémicas o enfermedades de base como deficiencias vitamínicas, enfermedades gastrointestinales o deficiencias inmunitarias (tabla 1) y, eventualmente, tratarlas ${ }^{5,13}$.

Tabla 2

Tratamiento de la estomatitis aftosa recurrente

\begin{tabular}{|c|c|}
\hline Fármacos & Posología \\
\hline \multicolumn{2}{|l|}{ Tópicos } \\
\hline Clorhexidina al $0,12 \%$ en enjuagues o gel & Tres veces al día hasta la desaparición de las úlceras \\
\hline Triclosán en gel o enjuagues & Tres veces al día hasta la desaparición de las úlceras \\
\hline Amlexanox al $5 \%$ (parches orales, tabletas o pastas adhesivas) & Dos a 4 veces al día hasta la desaparición de las úlceras \\
\hline Doxiciclina $100 \mathrm{mg}$ & Disolver en $10 \mathrm{ml}$ de agua, enjuagar 4 veces al día durante 3 días \\
\hline $\begin{array}{l}\text { Acetónido de triamcinolona (solución acuosa o en orabase) } \\
\text { en concentraciones de } 0,05-0,5 \%\end{array}$ & Aplicado de 3 a 10 veces al día durante 3-5 min, hasta la desaparición de las úlceras \\
\hline $\begin{array}{l}\text { Acetónido de fluocinolona (solución acuosa o en orabase) en } \\
\text { concentraciones de } 0,025-0,05 \%\end{array}$ & Aplicado de 3 a 10 veces al día durante 3-5 min, hasta la desaparición de las úlceras \\
\hline $\begin{array}{l}\text { Propionato de clobetasol (solución acuosa o en orabase) al } \\
0,025 \%\end{array}$ & Aplicado de 3 a 10 veces al día durante 3-5 min, hasta la desaparición de las úlceras \\
\hline \multicolumn{2}{|l|}{ Sistémicos } \\
\hline Prednisona & Dosis de $25 \mathrm{mg} /$ día al inicio, seguido de una reducción de la dosis durante los siguientes 2 meses \\
\hline Talidomida & Dosis de $50-100 \mathrm{mg} /$ día durante 2 a 3 meses \\
\hline Levamisol & Dosis de $150 \mathrm{mg}$ al día, 3 veces a la semana, durante 6 meses \\
\hline
\end{tabular}


Tabla 3

Síndromes asociados a ulceración oral

$\begin{array}{ll}\text { Síndrome PFAPA } & \begin{array}{l}\text { Acrónimo de las palabras en inglés periodic fever, aphtae, pharyngitis y cervical adenopathies (fiebre periódica, aftas, faringitis y } \\ \text { adenopatías cervicales), es una de las causas de fiebre periódica en pediatría y se caracteriza por episodios febriles recurrentes } \\ \text { (cada } 3 \text { a } 6 \text { semanas), acompañados en el } 65-80 \% \text { de los casos de faringitis, aftas bucales y adenopatías cervicales }{ }^{22}\end{array} \\ \text { Síndrome de Behçet } & \begin{array}{l}\text { Trastorno sistémico inflamatorio, multigenético, de etiología desconocida. Las características clínicas incluyen úlceras orales y } \\ \text { genitales, inflamación ocular, lesiones en la piel, así como manifestaciones articulares, vasculares, neurológicas, pulmonares, } \\ \text { gastrointestinales y genitourinarias }\end{array} \\ \text { Síndrome de Sweet } & \begin{array}{l}\text { Trastorno caracterizado por fiebre, lesiones en piel de carácter eritematoso infiltrativo, leucocitosis con neutrofilia e infiltración } \\ \text { densa de la dermis por neutrófilos maduros }\end{array}\end{array}$

El tratamiento a prescribir (que sería extrapolable en buena medida a otros tipos de úlceras, como las descritas con anterioridad) lo condicionan la gravedad y sintomatología dolorosa de la enfermedad, la frecuencia de los episodios con úlceras y la tolerancia del paciente a la medicación (tabla 2$)^{13}$. Frecuentemente se empieza con tratamiento tópico; la primera línea de tratamiento incluye antisépticos y medicamentos antiinflamatorios/analgésicos. Es muy útil la clorhexidina al $0,12 \%$ en enjuagues o gel. La duración del tratamiento se prolongará el tiempo necesario, hasta la resolución de las lesiones (suele ser unos días). El triclosán también puede ser utilizado en gel o enjuagues, ofreciendo efecto analgésico, antiinflamatorio y antiséptico ${ }^{13}$. Algunos autores proponen el empleo de amlexanox al $5 \%$ (parches orales, tabletas o pastas adhesivas) como tratamiento tópico de las úlceras. Se trata de un fármaco antiinflamatorio y antialérgico, que inhibe la formación y liberación de histamina y leucotrienos por parte de mastocitos, neutrófilos y células mononucleares en la zona afectada. Varios estudios han demostrado que acelera la cicatrización de las úlceras y resuelve el eritema, el dolor y el tamaño de las lesiones en la EAR ${ }^{13,14}$. Los antibióticos tópicos son otra opción de tratamiento. Entre ellos, enjuagues con hidrocloruro de tetraciclina, minociclina al $0,2 \%$ o doxiciclina en base adhesiva, que ayudan a mejorar la sintomatología dolorosa de los pacientes ${ }^{13,15}$. Los glucocorticoides tópicos son muy útiles en el tratamiento de la EAR. Los más empleados, en orden de menor a mayor potencia, son el acetónido de triamcinolona, el acetónido de fluocinolona y el propionato de clobetasol, en proporción adecuada, dependiendo de la gravedad de las lesiones ${ }^{13}$. Estos 3 medicamentos pueden ser administrados como pomadas en excipiente adhesivo, cuando las lesiones son localizadas, o en enjuagues en solución acuosa o hidroalcohólica cuando las lesiones son difusas o muy numerosas ${ }^{16}$. El acetónido de triamcinolona está indicado particularmente en pacientes con erosiones pequeñas y moderadas. El acetónido de fluocinolona brinda una potencia media-alta y se utiliza en pacientes con lesiones más agresivas. Por último, el propionato de clobetasol se reserva para las lesiones más importantes. En este contexto, se considera una alternativa previa a prescribir glucocorticoides sistémicos. Para ayudar a la curación se aconseja mantener la medicación en contacto directo con la lesión el mayor tiempo posible y no comer ni beber durante los 20 min posteriores a la aplicación del fármaco, evitando tocar la zona tratada. Si el medicamento es administrado en forma de enjuagues, se debe estar el tiempo indicado sin $\operatorname{tragar}^{13}$. Otra opción terapéutica de la EAR es el tratamiento sistémico, cuando el tratamiento tópico no es efectivo debido al grado de intensidad de las lesiones. Los glucocorticoides son la primera elección. La prednisona se ha utilizado, consiguiendo como resultado la desaparición del dolor y la reepitelización de las lesiones durante el primer mes de tratamiento. El tratamiento a largo plazo con glucocorticoides puede tener efectos adversos. Los inmunomoduladores como la talidomida también son utilizados como tratamiento para la EAR, obteniendo buenos resultados en la remisión de las lesiones, pero con muchos efectos adversos como teratogenicidad, polineuropatías, somnolencia, estreñimiento, aumento del apetito, náuseas y dolor estomacal $^{13}$.
En el caso de úlceras asociadas a otros síndromes (tabla 3), se adoptarán medidas (tratamiento tópico) que alivien la sintomatología y se derivará a los pacientes para completar su estudio.

En resumen, las úlceras orales se presentan con gran frecuencia en la población y pueden ser la primera señal de enfermedad sistémica. Estas lesiones pueden llegar a afectar la vida cotidiana del paciente, ya que al ser muy dolorosas dificultarán su normal masticación, deglución y habla, siendo por esto un motivo frecuente de consulta. Debido a que pueden tener múltiples causas es muy importante poner énfasis en la realización de una correcta historia clínica del paciente, tomando en cuenta las características clínicas de la úlcera así como su localización y tiempo de evolución. De esta manera se logrará realizar un buen diagnóstico diferencial, que posibilite al paciente un tratamiento efectivo.

\section{Conflicto de intereses}

Los autores declaran no tener ningún conflicto de intereses.

\section{Bibliografía}

1. Del Olmo-López J, Pretel-Irazabal M, España-Alonso A. Úlceras orales. Piel. 2006;21:92-100.

2. Scully C, Felix DH. Oral medicine-update for the dental practitioner. Aphthous and other common ulcers. Br Dent J. 2005;199:259-64.

3. Bascones-Martínez A, Figuero-Ruiz E, Carlos Esparza-Gómez G. Úlceras orales. Med Clin (Barc). 2005;125:590-7.

4. Parent D, Vaillant L. Aftas, aftosis, enfermedad de Behçet. EMC - Dermatología. 2008;42:1-20.

5. Ortiz Vega AP, Chimenos Küstner E. Diagnóstico diferencial de las úlceras orales. Piel. 2002;17:119-27

6. Scully C, Porter S. Oral mucosal disease: Recurrent aphthous stomatitis. Br J Oral Maxillofac Surg. 2008;46:198-206.

7. Siqueira CS, Saturno JL, de Sousa SCOM, da Silveira FRX. Diagnostic approaches in unsuspected oral lesions of syphilis. Oral Surg Oral Med Oral Pathol Oral Radiol. 2012;114:e62.

8. Bascones Martínez A, Valero Marugán A, Carrillo de Albornoz Sainz A, Encinas Bascones A. Afectación oral de las enfermedades comunes en la infancia con carácter exantemático. Av Odontoestomatol. 2006:22:163-70.

9. Muñoz-Corcuera M, Esparza-Gómez G, González-Moles Ma, Bascones-Martínez A. Oral ulcers: Clinical aspects. A tool for dermatologists. Part II. Chronic ulcers. Clin Exp Dermatol. 2009;34:456-61.

10. Kamala R, Sinha A, Srivastava A, Srivastava S. Primary tuberculosis of the oral cavity. Indian J Dent Res. 2011;22:835-8.

11. Aoun N, El-Hajj G, el Toum S. Oral ulcer: An uncommon site in primary tuberculosis. Aust Dent J. 2015;60:119-22.

12. López-López J, Omaña-Cepeda C, Jané-Salas E. Precáncer y cáncer bucal. Med Clin (Barc). 2015, http://dx.doi.org/10.1016/j.medcli.2014.11.014.

13. Belenguer-Guallar I, Jiménez-Soriano Y, Claramunt-Lozano A. Treatment of recurrent aphthous stomatitis. A literature review. J Clin Exp Dent. 2014;6:e168-74.

14. Darshan DD, Kumar CNV, Kumar ADM, Manikantan NS, Balakrishnan D, Uthka MP. Clinical study to know the efficacy of Amlexanox 5\% with other topical antiseptic, analgesic and anesthetic agents in treating minor RAS. J Int Oral Health. 2014;6:5-11.

15. Vijayabala GS, Kalappanavar AN, Annigeri RG, Sudarshan R, Shettar SS. Single application of topical doxycycline hyclate in the management of recurrent aphthous stomatitis. Oral Surg Oral Med Oral Pathol Oral Radiol. 2013;116: 440-6.

16. Muñoz-Corcuera M, Esparza-Gómez G, González-Moles MA, Bascones-Martínez A. Oral ulcers: Clinical aspects. A tool for dermatologists. Part I. Acute ulcers. Clin Exp Dermatol. 2009;34:289-94. 
17. Chavan M, Jain H, Diwan N, Khedkar S, Shete A, Durkar S. Recurrent aphthous stomatitis: A review. J Oral Pathol Med. 2012;41:577-83.

18. Ślebioda Z, Szponar E, Kowalska A. Recurrent aphthous stomatitis: Genetic aspects of etiology. Postepy Dermatol Alergol. 2013;30:96-102.

19. Ślebioda Z, Szponar E, Kowalska A. Etiopathogenesis of recurrent aphthous stomatitis and the role of immunologic aspects: literature review. Arch Immunol Ther Exp (Warsz). 2014;62:205-15.

20. Huling LB, Baccaglini L, Choquette L, Feinn RS, Lalla RV. Effect of stressful life events on the onset and duration of recurrent aphthous stomatitis. J Oral Pathol Med. 2012;41:149-52.
21. Adler I, Muiño A, Aguas S, Harada L, Diaz M, Lence A, et al. Helicobacter pylori and oral pathology: Relationship with the gastric infection. World J Gastroenterol. 2014;20:9922-35.

22. André S, Vales F, Cardoso E, Santos M. Síndrome PFAPA. Acta Otorrinolaringo Esp. 2009;60:208-9

23. Saleh Z, Arayssi T. Update on the therapy of Behçet disease. Ther Adv Chronic Dis. 2014:5:112-34.

24. Kaszewski S, Czajkowski R, Protas-Drozd F, Placek W, Jakubowski S. Sweet's syndrome with idiopathic thrombocythemia. Postep Derm Alergol. 2014;31: 47-52.

Cómo citar este artículo: Schemel-Suárez M, et al. Úlceras orales: diagnóstico diferencial y tratamiento. Med Clin (Barc). 2015. 